\title{
Whole-genome sequencing of a monozygotic twin discordant for systemic lupus erythematosus
}

\author{
FEI CHEN ${ }^{1 *}, \mathrm{ZHEN} \mathrm{LI}^{2 *}, \mathrm{RONG} \mathrm{LI}^{1}$ and YUNLONG LI ${ }^{1}$ \\ ${ }^{1}$ Department of Clinical Medicine, Affiliated Hospital of Kunming University of Science and Technology \\ (The First People's Hospital of Yunnan Province), Kunming University of Science and Technology, \\ Kunming, Yunnan 650500; ${ }^{2}$ Department of Breast Surgery, Yunnan Tumor Hospital, \\ The Third Affiliated Hospital of Kunming Medical University, Kunming, Yunnan 650118, P.R. China
}

Received November 15, 2017; Accepted March 5, 2018

DOI: $10.3892 / \mathrm{mmr} .2018 .8912$

\begin{abstract}
Systemic lupus erythematosus (SLE) is an autoimmune disease, and its genetic causes remain to be fully elucidated. Previous studies have identified several susceptibility genes for SLE, such as deoxyribonuclease 1-like 3. In the present study, whole-genome sequencing (30X coverage) was performed on the leukocytes of a monozygotic twin discordant for SLE to assess the potential association of de novo variants and copy number variations (CNVs) with the susceptibility to SLE. After analyzing the genomic data, 8 putative discordant exonic variants between the twins were selected. However, the 8 variants that were chosen for validation with Sanger sequencing exhibited no discrepancy in the leukocytes from the twins. Of note, CNV alterations in genes of SLE-associated pathways were identified between the twins, which may be linked with the phenotype of the monozygotic twin discordant for SLE. The above results suggest that genomic sequences of leukocytes in the monozygotic twins may exhibit a rare difference, and that CNV changes may be associated with phenotype differences in the twin discordant for SLE.
\end{abstract}

\section{Introduction}

Systemic lupus erythematosus (SLE) is an autoimmune disease $(1,2)$. The pathological mechanism comprises the attack of numerous parts of healthy tissues in the body by the patients' own immune system (3). SLE patients present

Correspondenceto: DrYunlongLi,Department ofClinicalMedicine, Affiliated Hospital of Kunming University of Science and Technology (The First People's Hospital of Yunnan Province), Kunming University of Science and Technology, 727 Jing Ming Nan Road, Kunming, Yunnan 650500, P.R. China

E-mail: yunlongli.km@gmail.com

*Contributed equally

Key words: systemic lupus erythematosus, monozygotic twin, variants, copy number variations with painful and swollen joints, fever and chest pain (4). The exact causes of SLE have remained to be elucidated; however, hormonal, environmental and genetic factors are thought to be associated with the causes of SLE (5). At present, no cure is available for SLE, and the life expectancy of SLE patients is relatively short (6). Therefore, further research is required in the field of SLE. Understanding the exact causes of SLE will facilitate the therapy and drug design for SLE.

In the past several years, numerous susceptibility genes for SLE have been discovered, including interferon regulatory factor (IRF)5, signal transducer and activator of transcription 4, IKAROS family zinc finger 1, ETS proto-oncogene 1 and deoxyribonuclease 1-like 3 (7-11). Although the exact functional association between these genes and SLE require further investigation, these results indicate a link of genetic factors with SLE. Comparing the genomes of monozygotic twins discordant for phenotypes is a tool to identify possible mutations and copy number variations (CNVs) causing these discordant phenotypes (12-14). Kondo et al (15) studied a monozygotic twin discordant for Van der Woude and popliteal pterygium syndromes. They identified genetic differences in the IRF6 gene that are associated with the phenotype discordance for the monozygotic twin. In the present study, whole-genome sequencing (WGS), a widely used research tool for studying genetic disease (16-18), was applied to assess a monozygotic twin discordant for SLE to identify the possibly responsible mutation(s).

In the present study, a monozygotic twin discordant for SLE was assessed by using WGS. Although the putative discordant exonic variants between the twins were selected from the WGS data, it was not possible to further verify these variants by conventional Sanger sequencing. Of note, CNVs changes in SLE pathway genes were detected in the twin discordant for SLE. Therefore, CNVs changes in SLE genes may be associated with the pathology of the twin discordant for SLE.

\section{Materials and methods}

Patients and genomic DNA isolation. Whole blood samples of twin A and twin B were obtained at the First People's Hospital of Yunnan Province (Kunming, China) during their visit to the clinical center in August 2016. The affected individual 
(twin A) was diagnosed with SLE. The parents of the twins are healthy.

The present study was approved by the Ethics Committee of the First People's Hospital of Yunnan Province (Affiliated Hospital of Kunming University of Science and Technology). Written informed consent was obtained from the participants. Genomic DNA from the whole blood samples were extracted using the E.Z.N.A. ${ }^{\circledR}$ Blood DNA Kit (cat. no. D3392-02; Omega Bio-Tek, Inc., Norcross, GA, USA) according to the manufacturer's protocol.

WGS and single nucleotide variant (SNV)/insertions or deletions (indel) and CNV analysis. WGS was performed on the genomic DNA of the leukocytes from each of the twins by using the HiSeq X Ten platform (Macrogen, Inc., Seoul, South Korea). All of the experiments were performed by using TruSeq Nano DNA kit (cat. no. 20015965; Illumina, San Diego, CA, USA) based on the Illumina TruSeq Nano DNA library preparation guide (Illumina). In brief, genomic DNA was fragmented by Covaris sonicator system to obtain 300-400 bp fragments. The DNA fragments were converted into blunt ends using the End Repair mix (Illumina). A single 'A' nucleotide was added to the 3 ' ends of the blunted fragments to prevent them from ligating to one another during the adapter ligation reaction. The ends of the DNA fragments were ligated to multiple indexing adapters. The DNA fragments were then sequenced using a HiSeq X Ten sequencer (Macrogen, Inc.).

Candidate SNVs, indels and CNVs were extracted and annotated by software including Isaac Aligner (19), Isaac Variant Caller (19), SnpEff (20), Control-FREEC (21) and Manta (22). The detailed steps of analyzing WGS data are displayed in Table I. In general, the non-synonymous, splicing, stop gained, stop loss and frame shift variants were extracted from the total variants. The SNVs/indels were further filtered by using the dbSNP (23), 1000 genome (24) and ESP6500 databases (http://evs.gs.washington.edu/EVS/) with a threshold of minor allele frequency $(\mathrm{MAF})<0.01$. The candidate discordant SNVs/indels between the twins were identified by comparing the above variants of twin A (the affected individual) with those of twin B. The discordant SNVs/indels observed in twin A and twin B were determined as a candidate discordant $\mathrm{SNV}$ /indels set. These variants were further filtered by using SIFT score $<0.05(25)$, Polyphen-2 score $>0.85$ (26) and other criteria [insufficient coverage $(<5)$ and obvious false-positive variants]. Integrative Genomics Viewer software $(27,28)$ was used to exclude the obvious false-positive variants by visualization of alignment data from the two twins. After the above filtrations, the putative discordant SNVs/indels were selected out and validated by conventional Sanger sequencing.

In the CNV analysis, the CNVs between twin A and twin $\mathrm{B}$ were compared. The discordant $\mathrm{CNV}$ s between twin $\mathrm{A}$ and twin B were respectively subjected to Kyoto Encyclopedia of Genes and Genomes (KEGG) pathway enrichment analysis by using PERL and the R programming language. R's phyper was used to calculate the P-value in the KEGG pathway enrichment analysis.

Polymerase chain reaction (PCR) amplification and sequencing. The fragments of candidate genes harboring the discordant SNVs/indels (Table I) were amplified using PCR. The primers used for amplification and sequencing of zinc finger protein 595, ankyrin repeat domain 20 family member (ANKRD20)A2, ANKRD20A4, ArfGAP with GTPase domain, Speedy/RINGO cell cycle regulator family member E2B, $\gamma$-glutamyltransferase 1 (GGT1) and eukaryotic translation initiation factor 3 gene fragments were obtained from the Beijing Genomics Institute (Shenzhen, China). The sequences of the primers are available on request. Amplification of the fragments was performed in a volume of $25 \mu \mathrm{l}$ containing $30 \mathrm{ng}$ genomic DNA, $50 \mu \mathrm{M}$ deoxynucleoside triphosphate, 10X LA Taq ${ }^{\mathrm{TM}}$ PCR buffer, 2.5 units of Takara LA Taq ${ }^{\mathrm{TM}}$ (Takara Bio Inc., Otsu, Japan) and $0.2 \mu \mathrm{M}$ of each forward and reverse primer. The PCR amplification for these gene fragments was performed using a denaturation step of $94^{\circ} \mathrm{C}$ for $5 \mathrm{~min}$, followed by 35 cycles of denaturation at $94^{\circ} \mathrm{C}$ for $30 \mathrm{sec}$, annealing at $60^{\circ} \mathrm{C}$ and extension at $72^{\circ} \mathrm{C}$ for $30 \mathrm{sec}$, and ended with a final extension step at $72^{\circ} \mathrm{C}$ for $7 \mathrm{~min}$.

The PCR products were purified using a Genomic DNA Purification kit (cat. no. DP204-02; Tiangen Biotech Co., Ltd., Tiangen, China) and were sequenced using sequencing primers (available upon request) and the Big Dye Terminator v.3.1 Cycle Sequencing kit (cat. no. 4337456; Applied Biosystems; Thermo Fisher Scientific, Inc., Waltham, MA, USA) on an ABI Prism 3730 DNA sequencer (Applied Biosystems; Thermo Fisher Scientific, Inc.). Sequences were compared between the two twins to verify the discordant variants.

\section{Results}

WGS. To identify a potential association of de novo variants and CNVs with SLE, WGS was performed on the DNA of leukocytes from the two twins. A total of 3,585,567 and $793,057,758$ variants were identified in twin A and B, respectively. The number of non-synonymous, splicing, stop gained, stop loss and frame shift variants in twin A and B was 22,120 and 22,125 , respectively. The above potentially functional variants were further filtered with the dbSNP (23), 1000 genome (24) and ESP6500 databases (http://evs.gs.washington .edu/EVS/), as well as SIFT score <0.05 (19) and Polyphen-2 score $>0.85$ (26), as presented in Table I. At last, 8 putative variants in 7 genes were finally selected out for subsequent Sanger sequencing (Table II). Of the 7 genes, only the GGT1 gene has records in the Online Mendelian Inheritance in Man database (29). The GGT1 gene has been reported to be associated with diseases, including glutathionuria and extrahepatic cholestasis. The 8 discordant variants were validated by conventional Sanger sequencing. None of the above discordant variants was successfully validated by Sanger sequencing (Fig. 1). To tell from the results, the discordant variants from the WGS may have exhibited minor differences between twin $\mathrm{A}$ and twin $\mathrm{B}$.

CNV analysis. As presented in Table I, twin A as well as twin $\mathrm{B}$ displayed changes in CNVs. The discordant CNVs in twin A and twin B were respectively subjected to KEGG pathway enrichment analysis. Of all discordant CNVs in twin A and twin $\mathrm{B}$, only the genes with discordant copy number losses in twin A (the affected individual) were significantly enriched in pathways including SLE, alcoholism and olfactory transduction, as 
Table I. Sequencing results and exclusion criteria for whole genome sequencing data analysis.

\begin{tabular}{lr}
\hline Parameter & Twin A \\
\hline Total reads & $782,904,696$ \\
Total yield (Mbp) & 117,435 \\
Mappable yield (Mbp) & 100,041 \\
Mappable mean depth (X) & 35.00 \\
SNVs and Indels & 118,958 \\
No. of total variants & 101,845 \\
No. of non-synonymous, splicing, stop gained, stop loss and frame shift variants & 35.60 \\
No. of variants after dbSNP, 1,000 genome and ESP6500 databases filtering & 22120 \\
(Minor allele frequency<0.01) & 234 \\
No. of variants in twin A but not in twin B and filtered by SIFT (<0.05), polyphen-2 & 22125 \\
(>0.85) and other criteria[insufficient coverage (<5) and obvious false-positive variants] & 234 \\
No. of putative discordant exonic variants chosen for Sanger sequencing (Table II) & 17 \\
No. of chosen putative discordant variants validated by Sanger sequencing & 8 \\
Copy no. variants & 0 \\
Gains (>2) & 390 \\
Losses $(<2)$ & 554 \\
\hline
\end{tabular}

Values are expressed as n unless otherwise specified. SNVs, single nucleotide variants; Indels, insertions or deletions.
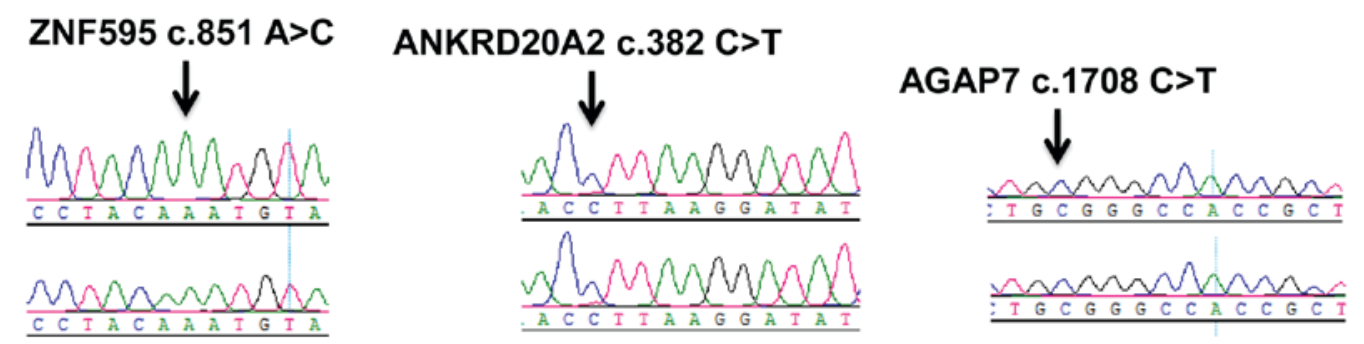

\section{SPDYE2L C.337 T>C}
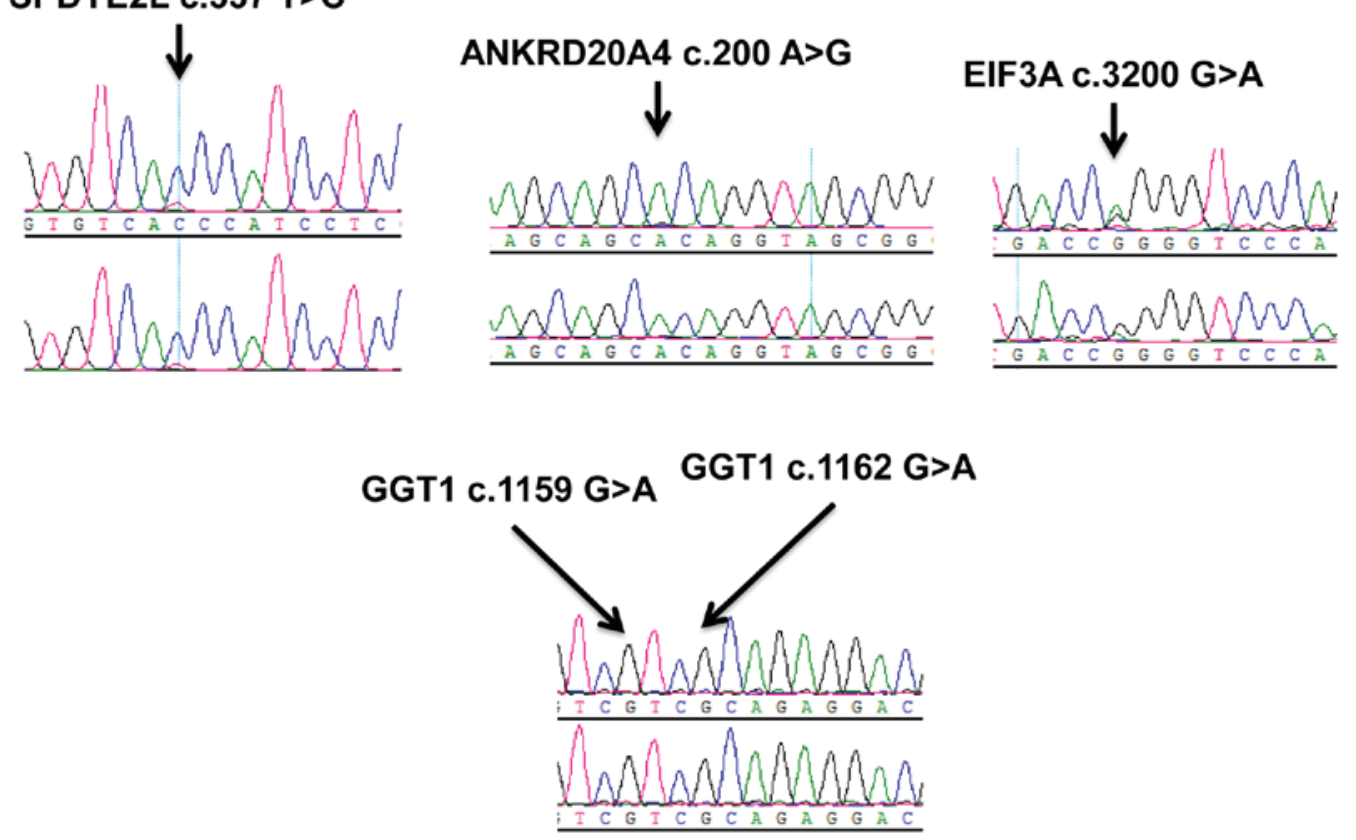

Figure 1. Sanger sequencing results of the 8 selected discordant variants between the monozygotic twins. The sequencing results of twin A (affected by systemic lupus erythematosus) and the discordant twin B are presented in the upper and lower electropherograms of each panel, respectively. ZNF595, zinc finger protein 595; ANKRD20, Ankyrin repeat domain 20 family; AGAP7, ArfGAP with GTPase domain, ankyrin repeat and PH domain 7; SPDYE2L, Speedy/RINGO cell cycle regulator family member E2B; EIF3A, eukaryotic translation initiation factor 3 subunit A; GGT1, $\gamma$-glutamyltransferase 1. 

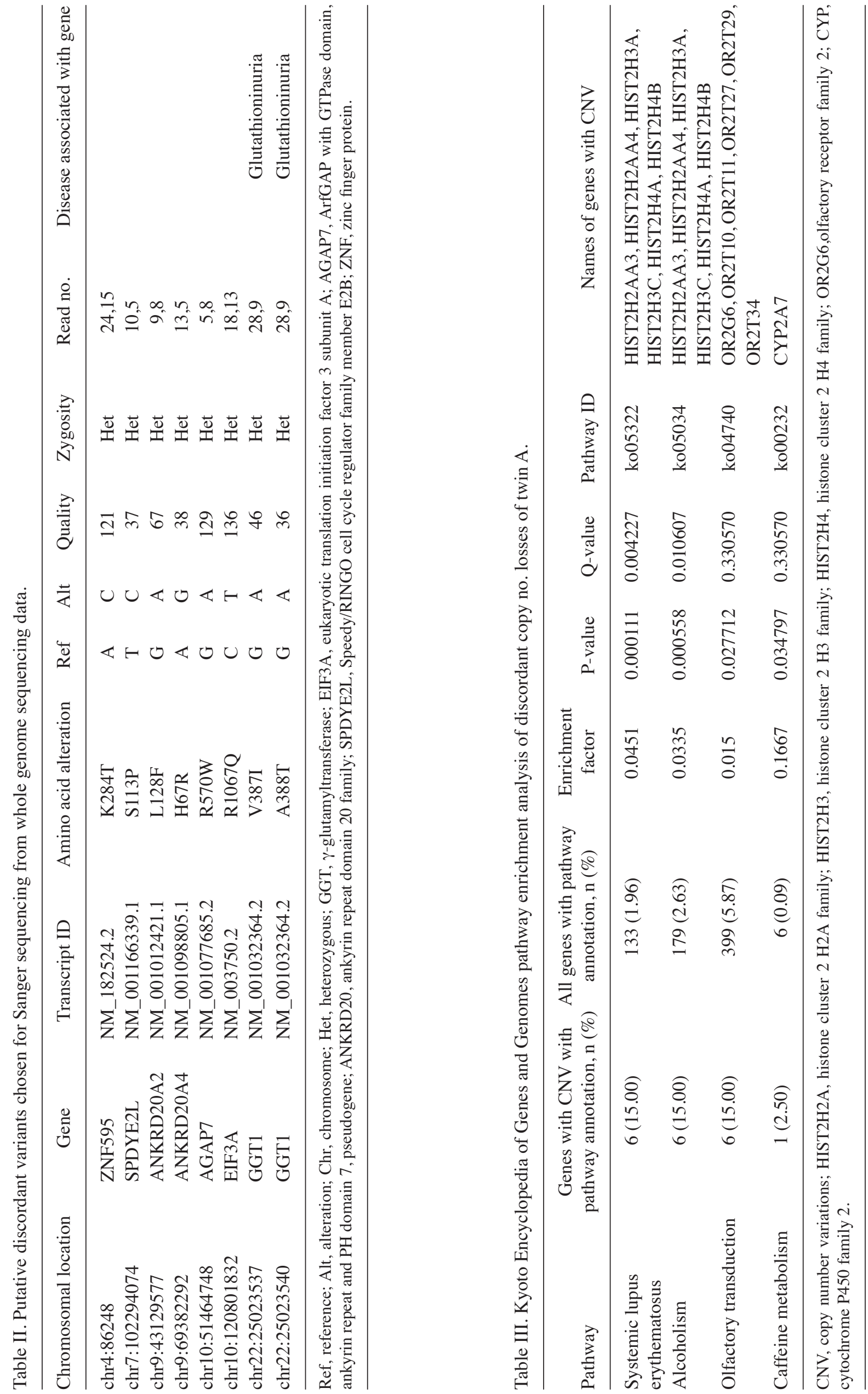
presented in Table III. Enriched SLE pathway genes with copy number losses in twin A were histone cluster $2 \mathrm{H} 2 \mathrm{~A}$ family member A3 (HIST2H2AA3), HIST2H2AA4, HIST2H3A, HIST2H3C, HIST2H4A and HIST2H4B. The results suggested that $\mathrm{CNV}$ changes may be associated with the phenotype of the monozygotic twin discordant for SLE.

\section{Discussion}

At present, studies assessing monozygotic twins discordant for SLE by using WGS are rare. Due to the possibility of the existence of de novo variants and alterations of CNVs in monozygotic twins $(13,15,18,30)$, the present study used WGS to explore the possible disease-causing variants which are responsible for the discordance in monozygotic twins regarding SLE. A total of 8 putative discordant variants in the DNA of leukocytes were selected out for validation by Sanger sequencing. However, no difference was identified in these variants between the monozygotic twins. Of note, copy numbers in certain SLE pathway genes exhibited alterations in monozygotic twins discordant for SLE.

The invalidation of the selected discordant variants in the present study was consistent with previous similar studies. The studies by Baranzini et al (31), Chaiyasap et al (32) and Solomon et al (33) did not identify any discordant variants in monozygotic twins discordant for multiple sclerosis, congenital heart defect or vertebral defects, anal atresia, cardiac defects, tracheo-esophageal fistula, renal anomalies and limb abnormalities, respectively. By contrast, Reumers et al (30) and Tang et al (18) identified discordant variants in the monozygotic twins discordant for schizophrenia. The results of these studies suggested that the de novo mutations in the monozygotic twin discordant for certain phenotypes may be specific for certain types of disease. Furthermore, as for the unsuccessful identification of discordant variants in the present study and certain previous studies, the following considerations were raised for using next-generation sequencing (NGS) to study monozygotic twins discordant for certain phenotypes: i) Although NGS technology is able to efficiently sequence a large amount of genes at a time, it also has high error rates (34). The discordant variants obtained by WGS in the present study may be due to sequencing errors. In addition, the average sequencing depth in the present study was $30 \mathrm{X}$, so that not all genomic regions were completely sequenced. It is possible that the sequencing coverage of the present study had no sufficient power to identify de novo variants linked with SLE. ii) DNA in leukocytes may not be an ideal sample for studying monozygotic twins discordant for phenotypes $(32,35)$. In pregnancy, most monozygotic twins are monochorionic. As they share the same blood circulation, it is possible that monozygotic twins share the same hematopoietic systems. Tissue samples displaying discordant phenotypes from monozygotic twins may be better for studying the discordant variants, since they are directly associated with the discordant phenotypes. iii) The analyzing tool and standard for WGS data used in the present study mainly focus on the functional variants, e.g. exonic missense variants. It did not sufficiently assess the variants in non-coding regions and may have missed the significant discordant variants of the monozygotic twins.

SLE is an autoimmune disease in humans. The mechanisms in monozygotic twins discordant for autoimmune disease still remain to be fully elucidated. As for SLE, Javierre et al (36) reported DNA methylation pattern changes in a study of monozygotic twins discordant for SLE. In the present study, CNVs changes of SLE pathway genes were identified in monozygotic twins discordant for SLE. The above results indicated that epigenetic changes and $\mathrm{CNV}$ changes in SLE pathway genes may be responsible for monozygotic twins discordant for SLE. The detailed mechanisms require further investigation.

The present study employed the WGS technique to reveal discordant variants and CNVs in monozygotic twins discordant for SLE. The results suggested that CNV changes may be associated with the occurrence of monozygotic twins discordant for SLE. The results of the present study will be helpful in the future analysis of mechanisms of monozygotic twins discordant for SLE.

\section{Acknowledgements}

Not applicable.

\section{Funding}

The present study was supported by the Foundation of Medical Discipline Leaders Program of the Health and Family Planning Commission of Yunnan Province (grant no. D-201668), the Personnel Training Project of Yunnan Province (grant no. 2017HB043), the National Natural Science Foundation of China (grant nos. 81560126 and 81460424), the Joint Special Research Funds of Kunming Medical University [grant no. 2017FE468(-010)] and the Innovation Team of Yunnan Province (grant no. 2017HC009).

\section{Availability of data and materials}

The analyzed data sets generated during the study and primer sequences are available from the corresponding author on reasonable request.

\section{Authors' contributions}

FC, ZL and RL performed the experiments and analyzed the data. ZL and YL wrote the manuscript and designed the study. The final version of the manuscript was read and approved by all authors.

\section{Ethical approval and consent to participate}

The present study was approved by the Ethics Committee of the First People's Hospital of Yunnan Province (Affiliated Hospital of Kunming University of Science and Technology). Written informed consent was from the participants.

\section{Consent for publication}

Not applicable.

\section{Competing interests}

The authors declare that they have no competing interests. 


\section{References}

1. Mirabelli G, Cannarile F, Bruni C, Vagelli R, De Luca R and Carli L: One year in review 2015: Systemic lupus erythematosus. Clin Exp Rheumatol 33: 414-425, 2015.

2. Petri M: Review of classification criteria for systemic lupus erythematosus. Rheum Dis Clin North Am 31: 245-254, vi, 2005.

3. Petri M: Epidemiology of systemic lupus erythematosus. Best Pract Res Clin Rheumatol, 16: 847-858, 2002.

4. Ahmad YA and Bruce IN: Genetic epidemiology: Systemic lupus erythematosus. Arthritis Res 3: 331-336, 2001.

5. Mak A and Tay SH: Environmental factors, toxicants and systemic lupus erythematosus. Int J Mol Sci 15: 16043-16056, 2014.

6. Mok CC, Kwok RC and Yip PS: Effect of renal disease on the standardized mortality ratio and life expectancy of patients with systemic lupus erythematosus. Arthritis Rheum 65: 2154-2160, 2013.

7. Bodaño A, Amarelo J, González A, Gómez-Reino JJ and Conde C: Novel DNASE I mutations related to systemic lupus erythematosus. Arthritis Rheum 50: 4070-4071, 2004.

8. Leng RX, Wang W, Cen H, Zhou M, Feng CC, Zhu Y, Yang XK, Yang M, Zhai Y, Li BZ, et al: Gene-gene and gene-sex epistatic interactions of MiR146a, IRF5, IKZF1, ETS1 and IL21 in systemic lupus erythematosus. PLoS One 7: e51090, 2012.

9. Wang C, Sandling JK, Hagberg N, Berggren O, Sigurdsson S, Karlberg O, Rönnblom L, Eloranta ML and Syvänen AC: Genome-wide profiling of target genes for the systemic lupus erythematosus-associated transcription factors IRF5 and STAT4. Ann Rheum Dis 72: 96-103, 2013.

10. Yang W, Shen N, Ye DQ, Liu Q, Zhang Y, Qian XX, Hirankarn N, Ying D, Pan HF, Mok CC, et al: Genome-wide association study in Asian populations identifies variants in ETS1 and WDFY4 associated with systemic lupus erythematosus. PLoS Genet 6: e1000841, 2010.

11. Remmers EF, Plenge RM, Lee AT, Graham RR, Hom G, Behrens TW, de Bakker PI, Le JM, Lee HS, Batliwalla F, et al: STAT4 and the risk of rheumatoid arthritis and systemic lupus erythematosus. N Engl J Med 357: 977-986, 2007.

12. Ketelaar ME, Hofstra EM and Hayden MR: What monozygotic twins discordant for phenotype illustrate about mechanisms influencing genetic forms of neurodegeneration. Clin Genet 81: 325-333, 2012.

13. Maiti S, Kumar KH, Castellani CA, O'Reilly R and Singh SM: Ontogenetic de novo copy number variations (CNVs) as a source of genetic individuality: Studies on two families with MZD twins for schizophrenia. PLoS One 6: e17125, 2011

14. Silva S, Martins Y, Matias A and Blickstein I: Why are monozygotic twins different? J Perinat Med 39: 195-202, 2011.

15. Kondo S, Schutte BC, Richardson RJ, Bjork BC, Knight AS, Watanabe Y, Howard E, de Lima RL, Daack-Hirsch S, Sander A, et al: Mutations in IRF6 cause Van der Woude and popliteal pterygium syndromes. Nat Genet 32: 285-289, 2002.

16. Sobreira NL, Cirulli ET, Avramopoulos D, Wohler E, Oswald GL, Stevens EL, Ge D, Shianna KV, Smith JP, Maia JM, et al: Whole-genome sequencing of a single proband together with linkage analysis identifies a Mendelian disease gene. PLoS Genet 6: e1000991, 2010.

17. Petersen BS, Spehlmann ME, Raedler A, Stade B, Thomsen I, Rabionet R, Rosenstiel P, Schreiber S and Franke A: Whole genome and exome sequencing of monozygotic twins discordant for Crohn's disease. BMC Genomics 15: 564, 2014.

18. Tang J, Fan Y, Li H, Xiang Q, Zhang DF, Li Z, He Y, Liao Y, Wang Y, He F, et al: Whole-genome sequencing of monozygotic twins discordant for schizophrenia indicates multiple genetic risk factors for schizophrenia. J Genet Genomics 44: 295-306, 2017.

19. Raczy C, Petrovski R, Saunders CT, Chorny I, Kruglyak S, Margulies EH, Chuang HY, Källberg M, Kumar SA, Liao A, et al: Isaac: Ultra-fast whole-genome secondary analysis on Illumina sequencing platforms. Bioinformatics 29: 2041-2043, 2013.
20. Cingolani P, Platts A, Wang le L, Coon M, Nguyen T, Wang L, Land SJ, Lu X and Ruden DM: A program for annotating and predicting the effects of single nucleotide polymorphisms, SnpEff: SNPs in the genome of Drosophila melanogaster strain w1118; iso-2; iso-3. Fly (Austin) 6: 80-92, 2012.

21. Boeva V, Popova T, Bleakley K, Chiche P, Cappo J, Schleiermacher G, Janoueix-Lerosey I, Delattre O and Barillot E: Control-FREEC: A tool for assessing copy number and allelic content using next-generation sequencing data. Bioinformatics 28: 423-425, 2012.

22. Chen X, Schulz-Trieglaff O, Shaw R, Barnes B, Schlesinger F, Källberg M, Cox AJ, Kruglyak S and Saunders CT: Manta: Rapid detection of structural variants and indels for germline and cancer sequencing applications. Bioinformatics 32: 1220-1222, 2016.

23. Smigielski EM, Sirotkin K, Ward M and Sherry ST: DbSNP: A database of single nucleotide polymorphisms. Nucleic Acids Res 28: 352-355, 2000 .

24. 1000 Genomes Project Consortium, Auton A, Brooks LD, Durbin RM, Garrison EP, Kang HM, Korbel JO, Marchini JL, McCarthy S, McVean GA and Abecasis GR: A global reference for human genetic variation. Nature 526: 68-74, 2015.

25. Ng PC and Henikoff S: SIFT: Predicting amino acid changes that affect protein function. Nucleic Acids Res 31: 3812-3814, 2003.

26. Adzhubei IA, Schmidt S, Peshkin L, Ramensky VE, Gerasimova A, Bork P, Kondrashov AS and Sunyaev SR: A method and server for predicting damaging missense mutations. Nat Methods 7: 248-249, 2010.

27. Robinson JT, Thorvaldsdóttir H, Winckler W, Guttman M, Lander ES, Getz G and Mesirov JP: Integrative genomics viewer. Nat Biotechnol 29: 24-26, 2011.

28. Thorvaldsdottir H, Robinson JT and Mesirov JP: Integrative Genomics Viewer (IGV): High-performance genomics data visualization and exploration. Brief Bioinform 14: 178-192, 2013.

29. Amberger JS, Bocchini CA, Schiettecatte F, Scott AF and Hamosh A: OMIM.org: Online Mendelian Inheritance in Man $(\mathrm{OMIM} \AA)$, an online catalog of human genes and genetic disorders. Nucleic Acids Res 43: D789-D798, 2015.

30. Reumers J, De Rijk P, Zhao H, Liekens A, Smeets D, Cleary J, Van Loo P, Van Den Bossche M, Catthoor K, Sabbe B, et al: Optimized filtering reduces the error rate in detecting genomic variants by short-read sequencing. Nat Biotechnol 30: 61-68, 2011.

31. Baranzini SE, Mudge J, van Velkinburgh JC, Khankhanian P, Khrebtukova I, Miller NA, Zhang L, Farmer AD, Bell CJ, Kim RW, et al: Genome, epigenome and RNA sequences of monozygotic twins discordant for multiple sclerosis. Nature 464: 1351-1356, 2010.

32. Chaiyasap P, Kulawonganunchai S, Srichomthong C, Tongsima S, Suphapeetiporn K and Shotelersuk V: Whole genome and exome sequencing of monozygotic twins with trisomy 21 , discordant for a congenital heart defect and epilepsy. PLoS One 9: e100191, 2014.

33. Solomon BD, Pineda-Alvarez DE, Hadley DW, Hansen NF, Kamat A, Donovan FX, Chandrasekharappa SC, Hong SK, Roessler E and Mullikin JC; NISC Comparative Sequencing Program: Exome sequencing and high-density microarray testing in monozygotic twin pairs discordant for features of VACTERL association. Mol Syndromol 4: 27-31, 2013.

34. Nielsen R, Paul JS, Albrechtsen A and Song YS: Genotype and SNP calling from next-generation sequencing data. Nat Rev Genet 12: 443-451, 2011.

35. Erlich Y: Blood ties: Chimerism can mask twin discordance in high-throughput sequencing. Twin Res Hum Genet 14: 137-143, 2011.

36. Javierre BM, Fernandez AF, Richter J, Al-Shahrour F, Martin-Subero JI, Rodriguez-Ubreva J, Berdasco M, Fraga MF, O'Hanlon TP, Rider LG, et al: Changes in the pattern of DNA methylation associate with twin discordance in systemic lupus erythematosus. Genome Res 20: 170-179, 2010. 